\title{
La Section d'Or y Juan Gris: Un camino hacia un cubismo espiritual
}

\author{
The Section d'Or and Juan Gris: A way towards the \\ spiritual Cubism
}

\author{
Iñigo SARRIUGARTE GÓMEZ \\ Universidad del País Vasco
}

Recibido: 16-XI-2015

Aceptado: 28-III-2016

Resumen: El artista de origen madrileño Juan Gris tuvo la oportunidad de ser testigo directo de la gestación del cubismo a raíz de su estancia en 1906 en la capital francesa. A partir de aquí, comienza a relacionarse con distintos pintores y críticos vanguardistas, que le ofertarán nuevas posibilidades aperturistas para su posterior involucración cubista. El año 1912 marca su definitiva integración bajo la estela pictórica del cubismo sintético, destacando igualmente su relación con el grupo de Puteaux. Los encuentros con esta agrupación cubista le derivarán hacia postulados caracterizados por la aplicación de enrejados, que eran formulados bajo premisas matemáticas, así como un interés manifiesto por la sección aurea, pero sin ser olvidada por parte del artista la evocación de un discurso espiritual adecuado a las necesidades de su tiempo.

Palabras clave: Juan Gris, Cubismo sintético, Proporción aurea, Section d'Or.

Aвstract: The artist Juan Gris had the opportunity to see the beginning of Cubism, staying in 1906 in the French capital. From here, he starts to interact with other avant-garde painters and critics who offered him new possibilities for a Cubist involvement. The year 1912 marks his integration in the pictorial trend of Synthetic Cubism, also standing out his relationship with the group of Puteaux. The encounters with this Cubist group derived him towards postulates characterized by the application of lattice-works, which were formulated under mathematical aspects and a manifest interest in the Golden Section, but without forgetting the spiritual discourse before the needs of his time.

Keywords: Juan Gris, Synthetic Cubism, Golden Ratio, Section d'Or. 


\section{DATOS BIOGRÁFICOS Y DESARRO- LLO PRODUCTIVO}

José Victoriano González-Pérez, conocido como Juan Gris (1887-1927) ${ }^{1}$, realiza estudios entre 1904 y 1906 en la Escuela de Artes e Industrias de Madrid, donde se aplica en matemáticas, física, ingeniería y metodología científica², así como en diversos campos de las artes, que se complementan en el taller del pintor paisajista y de género José Moreno Carbonero ${ }^{3}$.

Durante este último año se desplaza a París, donde le acoge su amigo Daniel Vázquez Díaz, instalándose en el 13 de la Rue Ravignan, en el edificio del Bateau Lavoir, donde también vive Picasso, asistiendo de primera mano a la génesis del cubismo. Juan Gris llega a París justo en el momento (otoño de 1906) en que Picasso va a comenzar a pintar Les demoiselles d'Avignon. Un año después conoce al crítico Maurice Raynal ${ }^{4}$, con quien mantendrá una sólida amistad, y al pintor Georges Braque. A partir de aquí, su círculo de amistades se expande a Guillaume Apollinaire, André Salmon y Max Jacob, entre otros muchos.

Será a partir de 1912 cuando finalmente se adentre en este estilo pictórico, pero bajo la estela del cubismo sintético. La atracción intelectual por el cubismo, la cercanía física a Picasso y Braque, así como sus contactos con

${ }^{1}$ ALBRIGHT-KNOX ART GALLERY, Painters of the Section D'Or: The Alternatives to Cubism. [Exhibition] September 27-October 22, Buffalo-New York, 1967, p. 24.

${ }^{2}$ Estos estudios de carácter técnico aportarán al artista una visible seguridad para adentrarse posteriormente en los entramados compositivos y formativos de la Section d'Or.

${ }^{3}$ Para más información sobre este artista, consultar el siguiente catálogo de exposición: AA.VV., José Moreno Carbonero: homenaje en el 150 aniversario de su nacimiento, 1858-1942, Málaga, 2008 y E. LAFUENTE FERRARI, José Moreno Carbonero, Málaga, 1967.

${ }^{4}$ Parte de la correspondencia entre Maurice Raynal y el pintor español se puede encontrar en J. GRIS, Correspondencia y escritos, Barcelona, 2008 (edición crítica y traducción del francés de María Dolores JiménezBlanco). el grupo cubista de Puteaux ${ }^{5}$ serán los acicates que le derivarán de manera firme hacia estos lenguajes. Su decidida apuesta por esta corriente vanguardista le permite firmar en 1912 un contrato con el marchante alemán Henry Kahnweiler, pudiendo llevar a cabo su primera exposición individual en la Galería Sagot. Más adelante expone sus trabajos en la colectiva titulada La Section d'Or en la Galería Boétie.

Un año más tarde decide pasar una temporada en Céret con Picasso, donde perfecciona el lenguaje del cubismo sintético. Por otra parte, su amistad con Picasso se irá enfriando paulatinamente a raíz de esta convivencia. También durante este año irrumpe con fuerza el color y desarrolla una técnica compositiva derivada de los papiers collés, con planos superpuestos que organizan estructuralmente el cuadro. Ese mismo año, en 1913, Guillaume Apollinaire publica Méditations Esthéthiques. Les peintres Cubistes, dedicándole un apartado, donde lo califica como "el hombre que ha meditado sobre todo lo moderno, (...) el artista pintor que no quiere concebir más que estructuras nuevas, que no querría dibujar ni pintar otra cosa que formas materialmente puras" ${ }^{\prime \prime}$.

A Gris le interesó especialmente el "método deductivo", basado en no crear a partir de una botella un cilindro, tal y como lo planteaba Cézanne, sino en un sentido inverso extraer de un cilindro una botella, procediendo de lo abstracto a lo figurativo, estrategia que se aprecia especialmente desde 1917, donde las propuestas lineales se simplifican. En este sentido, el artista afirma lo siguiente: "Trabajo con los elementos del espíritu, con la imaginación, intento concretar lo que es abstracto, voy de lo general a lo particular, lo que significa que parto de una abstracción para llegar a un hecho real. Mi

\footnotetext{
${ }^{5}$ El Groupe de Puteaux también es conocido como Section d'Or. Consultar A.M. PRECKLER, Historia del Arte Universal de los siglos XIX y XX, vol. 2, Madrid, 2003, p. 103.

${ }^{6}$ G. APOLLINAIRE, Méditations Esthéthiques. Les peintres Cubistes, Genève, 1950, p. 61.
} 
arte es un arte de síntesis, un arte deductivo como dice Raynal"7 . Igualmente, añade lo siguiente: "Mi método de trabajo es justamente el inverso. Es deductivo. No es el cuadro $X$ el que llega a coincidir con el asunto elegido, sino el asunto el que llega a coincidir con el cuadro. Digo que es deductivo porque las relaciones pictóricas entre las formas coloreadas me sugieren ciertas relaciones particulares entre elementos de una realidad imaginativa. La matemática pictórica me conduce a la física representativa. La cualidad o la dimensión de una forma o de un color me sugiere la denominación o el adjetivo de un objeto" $^{\prime \prime}$.

Durante los años de la contienda internacional la producción asume un colorido más frío, apagado y alejado de toda exaltación luminosa, aspectos que muestran los duros años del conflicto bélico, caso de $E l$ tablero de ajedrez (1917). Tal y como comenta Eugenio Carmona en relación con el escenario europeo: "El periodo bélico fue un momento de reajuste para el Movimiento Moderno y, especialmente, para las dos tendencias más influyentes en el contexto español: cubismo y futurismo. En el transcurso de la contienda aparecerían las recuperaciones clasicistas de Picasso y Severini, por elegir dos ejemplos significativos, y Juan Gris comenzaría su esforzada labor de equiparación entre el lenguaje cubista y la tradición clásica de la pintura francesa" ${ }^{\prime \prime}$.

Este último, más adelante, se alejará de la excesiva rigidez de los planteamien-

${ }^{7}$ J. GRIS, Op.cit., p. 461. Extraído de L'Esprit Nouveau, ํㅡㄴ 5, 1921, pp. 533-534.

8 ÍDEM, Op.cit., p. 462. Extraído de ÍDEM, "Notas sobre mi Pintura", Der Querschnitt, nº 1-2, 1923, pp. 7778.

${ }^{9}$ E. CARMONA, “Novecentismo y vanguardia en las artes plásticas españolas 1906-1926", en AA.VV. La generación del 14. Entre el Novecentismo y la Vanguardia (1906-1926), Madrid, 2002, p. 42. Este mismo autor cuenta con interesantes trabajos relacionados con la obra de Juan Gris, por ejemplo, Juan Gris, Madrid, 2001; El cubismo y sus entornos, Madrid, 2005; Los años cubistas, Madrid, 2007; "Juan Gris, elevado al cubo", Descubrir el arte, $\mathrm{n}^{-}$77, 2005, pp. 14-23; etc. tos geométricos para adentrarse en pautas más suavizadas, líricas y matizadas cromáticamente, retomando el color y una mayor complejidad compositiva a partir de 1918.

En 1921 Sergei Diaghilev invita a Gris a presentar un proyecto para la decoración de su ballet Cuadro flamenco, con música de Manuel de Falla. Gris se traslada a Montecarlo para discutir el proyecto, pero el empresario ruso cambia de opinión y se lo ofrece a Picasso; no obstante, solicita al pintor madrileño que elabore los retratos de las figuras principales del ballet. En definitiva, la colaboración con los Ballets Rusos de Diaguilev, entre 1921 y 1924, se presentó polémica y llena de obstáculos, debido a las diferencias estéticas que mantenía con el mecenas ruso. Este traspiés no impide que diseñe escenografías para otros dos ballets de Diaghilev: Les Tentations de la Bergère ou l'Amour Vainqueur, con música de Montéclair y coreografía de Nijinski, y La Colombe, esta vez con diálogos musicales compuestos por el joven Francis Poulenc $^{10}$. Finalmente, debido a la falta de entendimiento con Diaghilev en relación con sus bocetos para los distintos decorados, tomará la decisión de romper su asociación con éste.

En los últimos años de su vida, a partir de 1925, retoma materiales como el gouache y la acuarela, realizando igualmente algunas ilustraciones para libros, dibujos y grabados para publicaciones de vanguardia, a la vez que expone en diferentes galerías. De acuerdo con Gaya Nuño: "1925 arroja un descenso considerable en su producción pictórica, siendo acaso el año más débil desde 1911. Son cuadros de grandes, románticas, melan-

${ }^{10}$ Este pianista y compositor francés (1899-1963) tuvo en los comienzos de su carrera profesional una marcada influencia de Erik Satie, aunque después su estilo se encaminaría hacia el neoclasicismo e igualmente, después de su acercamiento al catolicismo, a la elaboración de composiciones sacras. Fue miembro del grupo Les Six, junto a otros compositores locales como Honegger y Tailleferre. Estuvo cerca de los planteamientos técnicos del dadaísmo, produciendo melodías que rompían los parámetros más ortodoxos de la música dirigida al gran público. 
cólicas figuras femeninas, el Pierrot con guitarra, también melancólico, más tal o cual figura de no bien digerido aire pompeyano"11 . En agosto de 1925 el artista visita con frecuencia la Exposition des arts décoratifs de París, en uno de cuyos pabellones, proyectado por Le Corbusier, se expone una obra suya.

Juan Gris no fue un artista versado en grandes apariciones estelares, su vida se caracterizó por ser recogida y mantenerse con discreción, pero por su sólida aportación al cubismo se le solicitó su opinión para revistas vanguardistas, como Dada y Valori Plastici. No obstante, entre sus principales textos relacionados con el cubismo, debemos citar Notas sobre mi pintura, publicado en la revista alemana Der Querschnitt, dirigida por A. Flechtheim (verano 1923), donde explica su método deductivo; y la conferencia dada en la Universidad de La Sorbona, con el título Sobre las posibilidades de la pintura (15 mayo 1924), donde se sintetizan las principales ideas estéticas del artista. En relación con la conferencia, Gaya Nuño resalta los siguientes aspectos: "es el contenido de esta conferencia universitaria el que provee a la obra de Juan Gris de un respaldo doctrinal de insuperable claridad expositiva, caso rarísimo en todo pintor anterior... El razonamiento del castellano sobre la naturaleza del objeto y de su representación, sobre los colores y la composición, sobre la pintura como síntesis y como arquitectura, es de una lucidez plena y en ocasiones implacable, hasta llegar a la clarísima definición final..." ${ }^{12}$.

Dentro de este apartado biográfico no debemos olvidar que en 1922 se inicia en la Logia Voltaire ${ }^{13}$, perteneciente al Grand Orient de France $^{14}$, donde no sólo acude de manera 23.

${ }^{11}$ J.A. GAYA NUÑO, Juan Gris, Barcelona, 1985, p.

${ }^{12}$ Ibídem, p. 168.

13 Para obtener más información consultar J. A. GARCÍA-DIEGO, Antonio Machado y Juan Gris. Dos artistas masones, Móstoles, 1990, pp. 359-361.

14 La información referente a esta federación de Obediencia masónica se puede encontrar en su página constante a sus tenidas, sino que comienza a desarrollar una labor activa, lo que en 1924 le elevara al grado de Compañero y, un año más tarde, al de Maestro Masón. Finalmente, el artista fallece un 11 de mayo de 1927.

\section{LOS PRIMEROS PASOS DE LA SECTION D'OR}

Durante el año 1912 las exposiciones cubistas se habían multiplicado de manera visible, junto con un aumento de los debates artísticos en torno a dicho movimiento ante la diversificación de las variadas tendencias que se estaban produciendo dentro del cubismo. Entre los principales difusores teóricos estaban Guillaume Apollinaire, André Salmon y Maurice Raynal, así como los artistas Le Fauconnier, Gleizes, Metzinger, Delaunay, los hermanos Duchamp y Léger, mientras Braque y Picasso decidieron ausentarse de manera permanente de estos encuentros. No obstante, serán especialmente Marcel Duchamp y sus hermanos Jacques Villon (siendo su verdadero nombre Gaston Duchamp) y Raymond Duchamp-Villon, quienes impulsaron de manera decidida los debates teóricos centrados en el desarrollo científico del cubismo, y su conexión con la abstracción. Estas temáticas atrajeron a artistas como Picabia, Kupka y Juan Gris, entre otros.

Como intento de proyección social, en otoño de 1912 se muestran públicamente como el Grupo de Puteaux, organizando en la galería $L a$ Boétie la exposición colectiva $L a$ Section d'Or, donde se podían observar propuestas inmersas dentro del lenguaje cubista, pero que abarcaban las distintas inquietudes de aquellos creadores, que querían alejarse de las auras centrípetas de Picasso y Braque, que evidentemente no tomaron parte en esta muestra. Fue una exposición que englobó más de ochenta trabajos de 31 artistas, fundamentalmente seguidores del cubismo. Entre éstos, los tres hermanos Du-

web: http://www.godf.org/, consultado el 15 de noviembre de 2015. 
champ, Juan Gris, Fernand Leger, Francis Picabia, Roger de la Fresnaye, Albert Gleizes, Auguste Herbin, André Lhote, Louis Marcoussis, Jean Metzinger, André Dunoyer de Segonzac, Oleksandr Archipenko, Marie Laurencin, Henry Valensi y František Kupka, entre otros. Su objetivo fue emplear parámetros matemáticos de la Section d'Or, lo que les permitía distanciarse del cubismo más ortodoxo, además de imprimir una orientación más teórica al cubismo, aludiendo a la cuestión de la armonía perfecta de la forma. Debemos recordar que desde mediados del siglo XIX había surgido un nuevo interés por esta medida geométrica, mediante la publicación Neue Lehre von den Proportionen des Menschlichen Körpers de Adolf Zeising en $1854^{15}$.

La exposición fue acompañada de la publicación Bulletin de La Section d'Or (Fig. 1) y de varias conferencias. Los participantes de la exposición habían mirado a las matemáticas, la trigonometría, la química, el psicoanálisis y la música, entre otras áreas del saber. Estos cubistas no sólo examinaron la proporción divina, sino también lo que Ozenfant solía definir como las constantes: “El estudio de los aspectos constantes y universales de las sensaciones, de sus cualidades intrínsecas, permite crear una estética sin que en ella tenga que intervenir el juicio de valor belleza"16.

La elección de este título fue llevada a cabo por Jacques Villon y corroborada por el resto de compañeros cubistas, ya que simbolizaba sus creencias en la tradición y el orden, para representar modelos y relaciones que se dan en la naturaleza. Incluso el término derivaba de otras manifestaciones que iban desde la revista Bandeaux d'or ${ }^{17}$ hasta la

${ }^{15}$ R. HERTZ-FISCHLER, Adolph Zeising, 1810-1876: The Life and Work of a German Intellectual, Ottawa, 2004.

${ }^{16}$ Citado en H. PIÑÓN, El formalismo esencial de la arquitectura moderna, Barcelona, 2008, p. 102. Sobre el tema de las constantes, también consultar, por ejemplo, A. OZENFANT, Mémoires 1886-1962, Paris, 1968, p. 128.

${ }^{17}$ Pierre Jean Jouve fundó en 1907 la revista literaria Bandeaux d'or, cuya dirección asumió desde su fecha

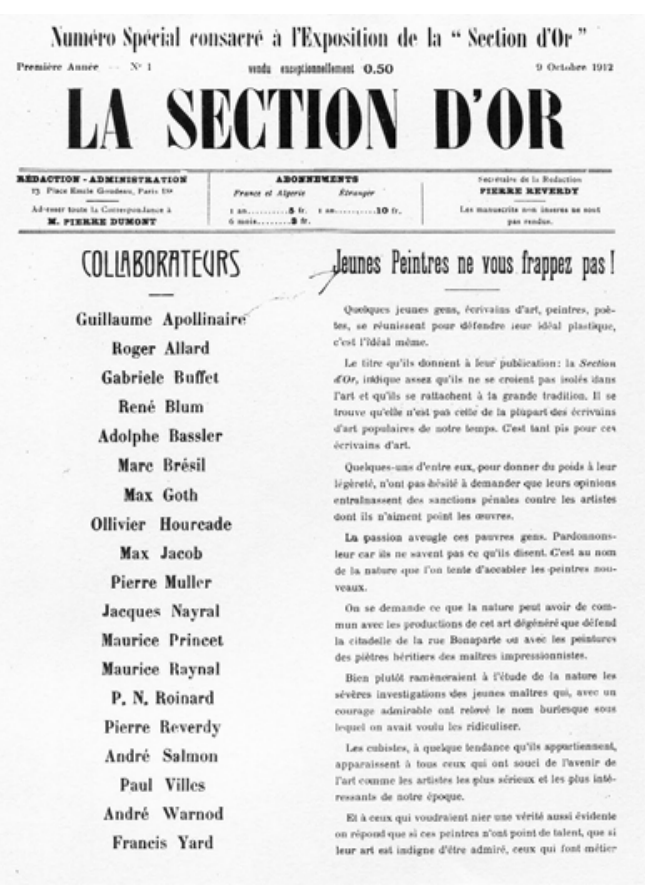

- Fig. 1. Bulletin de la Section d'Or, número especial, 9 Octubre de 1912. http://en.wikipedia.org/ wiki/Section_d\%27Or.

Societé Normande de la Peinture Moderne ${ }^{18}$, a la cual pertenecían los hermanos Duchamp desde 1909. En cualquier caso, la formación de la Section d'Or se constituye mediante la conjunción de dos grupos que confluyeron adoptando la misma ideología plástica; por un lado, los cubistas del Salon des Indépendants de 1911 (Gleizes, Metzinger, Delaunay, Léger y Le Fauconnier) y la Societé Normande de Peinture Moderne, que había surgido en Rouen a partir de 1909. Los miembros de ambas formaciones se habían unido en otoño de 1911 y se solían reunir "en el domicilio de Jacques Villon en Puteaux, en el estudio

de fundación hasta 1910. En relación con la deriva del término de la Section d'Or, remitirse a la siguiente página web: http://www.rij-rousseau.de/golden_section/ golden_section.html, consultado el 15 de noviembre de 2015.

${ }^{18}$ Sobre este colectivo de creadores franceses (escultores, pintores, músicos, poetas y críticos), relacionados con el postimpresionismo, fauvismo, cubismo y orfismo, debemos resaltar las siguientes publicaciones: C. STUART ROSS, The Société Normande de Peinture Moderne, Providence, 1970; D. COTTINGTON, Cubism and Its Histories, Manchester, 2004. 
de Albert Gleizes en Courbevoie, en un café de la Place de L'Alma o en las tertulias del escritor Paul Fort en la Closerie des Lilas en Montparnasse" $^{\prime 19}$. En dichos encuentros se trataban todo tipo de temáticas desde parámetros filosóficos a partir de Nietzsche y Bergson y la geometría no-euclidiana, hasta los últimos descubrimientos en ciencia y tecnología, así como la aplicación de las matemáticas en las artes.

Volviendo al título de la exposición, debemos comentar que este fue planteado por Jacques Villon, quien había leído Tratado de la Pintura de Leonardo da Vinci (redactado hacia finales del siglo $\mathrm{XV}$ y publicado en torno a 1680), en una traducción de 1910 realizada por Joséphin Peladan ${ }^{20}$, dando un gran valor y sentido místico a la Sección de Oro y a otras configuraciones geométricas. Villon había desarrollado su teoría del estudio de las pirámides, tomada de Leonardo da Vinci, sugiriendo durante estas reuniones el título de Section d'Or, que a su vez había sido extraído del tratado De Divina Proportione (escrito a finales del siglo XV) de Luca Pacioli. Debemos anotar el siguiente comentario de Jacques Villon: “De entrada, reclamo la paternidad de este título. En 1912, nuestras telas reunidas en una galería de la rue La Boétie formaron la primera exposición de la Section d'Or. En nuestras conversaciones hablába-

${ }^{19}$ P. ESTEBAN LEAL, “Una pasión contenida”, en AA.VV., Juan Gris. Pinturas y dibujos 1910-1927, Madrid, 2005, p. 34.

${ }^{20}$ Escritor y ocultista francés (1858-1918), que estudió con profundidad el Quattrocento italiano y especialmente la obra de Leonardo da Vinci. En 1888, junto a Stanislas de Guaita, funda la Orden Cabalística de la Rosa$\mathrm{Cruz}$, siendo algunos de sus miembros Erik Satie, Claude Debussy y el ocultista Papus. Tres años más tarde funda la Orden Rusacruz Católica y Estética del Templo y del Grial, para posteriormente organizar el primer $\mathrm{Sa}$ lon de la Rose Croix (abril de 1892) en la galería parisina Durand-Ruel. Su base estética se fundamenta en la defensa a ultranza de la belleza y la negación absoluta a la fealdad generada por el desarrollo del mundo moderno. En su producción literaria encontramos importantes trabajos de crítica de arte, así como las siguientes publicaciones: Franz Hals, Paris, 1912; La Doctrina de Dante, Paris, 1908; La última lección de Leonardo da Vinci, Paris, 1904; entre otras. mos mucho de la organización de la tela. La idea de que una tela debía ser razonada antes de ser pintada había calado hondo en nosotros. No sabíamos nada del problema de la sección de oro en las concepciones de los antiguos griegos. Yo había leído el Tratado de la Pintura de Leonardo y había visto la importancia que le daba. Pero fue sobre todo hablando como fijamos nuestras ideas sin saturarnos demasiado de ciencia" ${ }^{21}$.

El principal origen teórico de esta asignación se lo debemos al renacentista Luca Pacioli, en base a la relación $\mathrm{a} / \mathrm{b}$ resultante de la "división de una recta en media y extrema razón" ${ }^{22}$, desde donde se construye el nombre del título del libro De Divina Proportione (1509). Esta aplicación, ya utilizada anteriormente por Vitrubio y luego durante el Renacimiento, era el ideal de relación entre dos magnitudes, expresadas numéricamente, tal y como se puede observar en diferentes obras de arte. Uno de los artistas modernos que más se detuvo en esta premisa matemática fue el neoimpresionista George Seurat, que aplicaba en los laterales de sus lienzos dichas secciones áureas ${ }^{23}$.

La denominación de Pacioli se debe a que se generan evidentes correspondencias entre esta proporción y la divinidad misma. Pero será Leonardo da Vinci, ilustrador del

${ }^{21}$ P. ESTEBAN LEAL, Op. cit., pp. 34-35.

22 Sobre esta cuestión matemática se puede consultar M. LIVIO, The Golden Ratio: The Story of PHI, the World's Most Astonishing Number, New York, 2003, p. 3; S. OLSEN, The Golden Section: Nature's Greatest Secret, New York, 2006, p. 2; S. L. GRIFFING, The Golden Section: An Ancient Egyptian and Grecian Proportion, Philadelphia, 2007, p. 19.

${ }^{23}$ Para más datos, remitirse a L. HAUTECOEUR, Seurat, Milano, 1972, pp. 68-71. También se deberían destacar en esta misma línea las investigaciones realizadas por R. HERZ-FISCHLER, "An Examination of Claims Concerning Seurat and The Golden Number", Gazette des beaux-arts, 101, 1983, pp. 109-112; A. LHOTE, "Encyclopédie française", Arts et littératures dans la société contemporaine, vol. 16, part 1, 1935, p. 16.30-7, ill. pp. 16.30-6, 16.31-7 ; M. NEVEUX, Construction et proportion: apports germaniques dans une theorie de la peinture française de 1850 a 1950, Paris, 1990; M. F. ZIMMERMANN, Seurat and the Art Theory of His Time, Antwerp, 1991. 
libro de Pacioli, a quien se le debe la asignación del término con el cual se denomina esta misma proporción: sectio aure de donde proviene Section d'Or, que corresponde a un número irracional cuyo valor sería 1,618 (número de oro $)^{24}$. Siguiendo el entramado inicial de Pacioli, se llega al rectángulo áureo, del cual se obtiene una espiral ${ }^{25}$, cuya forma siempre se mantiene constante:

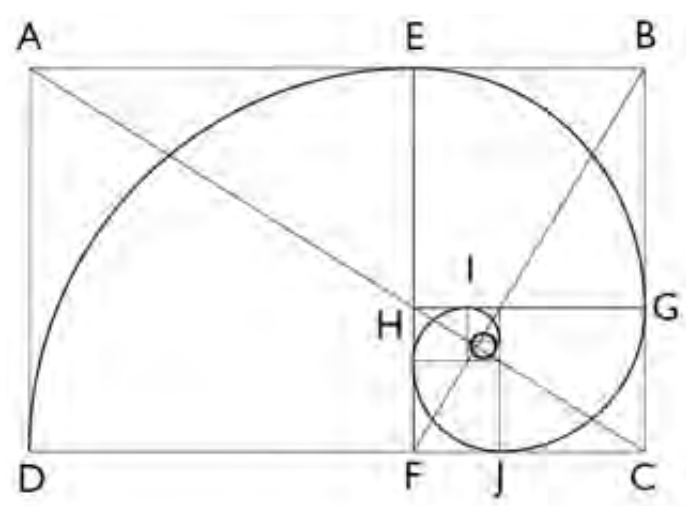

Dicha espiral se encuentra en la botáni$\mathrm{Ca}^{26}$, por ejemplo, en el perfil y en diversas secciones de las plantas, en los diagramas de crecimiento $^{27}$ y disposición de hojas y granos, así como en diferentes partes del cuerpo animal, caso de los cuernos de ciertos antílopes y cabras. Estos aspectos anteriores se relacionan directamente con la ley del crecimiento, lo que puede haber generado un interés por parte de la arquitectura y el arte. Por otro lado, el número áureo también se encuentra en el pentágono. Si la naturaleza orgánica se muestra bajo una simetría dinámica pentagonal, el ámbito inorgánico en base a su equilibrio perfecto aparenta totalmente una simetría hexagonal estática ${ }^{28}$. De

${ }^{24}$ Para más información consultar M. GHYKA, The geometry of Art and Life, New York, 1977, pp. 7-19.

${ }^{25}$ R. STASZKOW y R. BRADSHAW, The Mathematical Palette, Belmont, 2005, pp. 372-373.

${ }^{26}$ A. T. COOK, The Curves of Life, New York, 1979. (Obra original de 1914).

${ }^{27}$ Se pueden obtener más datos científicos sobre el tema del crecimiento en M. GHYKA, Estética de las proporciones en la naturaleza y el arte, Barcelona, 1983, pp. 122-152.

28 Véase G. DELEUZE, Difference and Repetition, London, 2007, p. 23. Igualmente, resulta de interés la hecho, este estudio sobre la simetría también había sido planteado por Juan Gris: "hay formas que tienen un eje de gravedad muy marcado y otras que lo tienen poco marcado. Las formas simétricas, en relación con su eje de gravedad, tienen más peso que las formas asimétricas y complicadas. Las figuras geométricas y las formas sometidas a un eje vertical tienen más gravedad que las formas cuyo eje no es vertical o no está acentuado" 29 .

Retomando nuevamente el sentido del pentagrama o pentalfa, este se asume, desde los pitagóricos hasta el Renacimiento, como un símbolo de salud, vida, perfección, belleza, e incluso representación del microcosmos, es decir, el hombre físico adaptado al macrocosmos (recuérdese el dibujo El hombre de Vitruvio de Leonardo da Vinci), siendo un símbolo de armonía entre el hombre y Universo. De igual manera, parece que Juan Gris busca estos conceptos de intemporalidad y lo absoluto en su pintura: "Hoy, evidentemente, me doy cuenta de que en sus comienzos el cubismo no era más que un modo nuevo de representación del mundo. Justamente por reacción contra los elementos fugitivos empleados por los impresionistas en sus representaciones, se ha querido buscar los elementos menos inestables en los objetos a representar. Y se eligió esa categoría de elementos que quedan en el espíritu por el conocimiento, y que no se modifican a cada hora" ${ }^{\prime 30}$.

Muchos de los participantes en la anterior exposición sólo mostraron una aplicación superficial del cubismo, en base a la fragmentación geométrica de la superficie pintada, siendo más el punto de unión la admiración por Cézanne que las propias formulacio-

consulta de la siguiente publicación: C. BOULEAU, Tramas. La geometría secreta de los pintores, Madrid, 1996; C. BONELL, La divina proporción. Las formas geométricas, Barcelona, 1994.

${ }^{29}$ J. GRIS, "Sobre las posibilidades de la pintura", en ÍDEM, De las posibilidades de la pintura y otros escritos, Barcelona, 1980, p. 42.

${ }^{30}$ ÍDEM, Correspondencia..., p. 401. Extraído de una respuesta dada a una encuesta de la revista Bulletin de la vie artistique, finales de 1924. 
nes matemáticas. Sin embargo, dentro de la muestra, se aprecia el interés de algunos de los miembros de este grupo por la abstracción matemática. En este sentido, cada artista tenía una serie de preocupaciones personales que de ninguna manera generaba una visión monolítica del cubismo; por ejemplo, para Kupka se debía promover la tendencia constructiva y una cercanía a la abstracción; Jacques Villon planteaba parámetros de composición y proporción de las partes del cuadro, dejando más de lado la referencia hacia la realidad representada; Marcel Duchamp se inclinó por los efectos cinéticos extraídos del cubismo mediante la angulación múltiple y la repetición de patrones simultáneos.

Muchos de estos elementos matemáticos no podían haber sido analizados y tratados si no hubiera sido por la presencia de Paul Sérusier ${ }^{31}$, miembro del Grupo de Pont-Aven y defensor de un lenguaje basado en la ciencia de los números y cuya aplicación práctica era la geometría. Estas teorías fueron redactadas en su libro $A B C$ de la Peinture (redactado en 1909, aunque publicado en 1921). Pero, especialmente, debemos anotar el nombre del matemático Maurice Princet ${ }^{32}$. En este sentido, tanto Juan Gris como Jean Metzinger desarrollaron un estudio de la geometría bajo la dirección de Princet con el propósito de explorar la aplicación de la geometría noeuclidiana y una cuarta dimensión para una pintura cubista. Tal y como afirma Metzinger: "Maurice Princet me dijo frente a Juan Gris que quería.......iniciarnos en las geometrías no-euclidianas, pidiéndonos crear

31 Para más información remitirse a R.V. WEST, Painters of the Section d'or: the alternatives to cubism, Buffalo, 1967 .

${ }^{32}$ Este matemático francés (1875-1973) tuvo un papel relevante en la evolución del cubismo, siendo incluso conocido como el mathématicien du cubisme. Mantuvo estrechos contactos con Picasso, Apollinaire, Metzinger, Max Jacob y Marcel Duchamp, entre otros. Introdujo el trabajo del matemático Henri Poincaré y las teorías de la Cuarta Dimensión a un nutrido grupo de artistas. Resulta interesante consultar T. ROBBIN, Shadows of Reality, The Fourth Dimension in Relativity, Cubism, and Modern Thought, New Haven, 2006; M. DÉCIMO, Maurice Princet, Le Mathématicien du Cubisme, Paris, 2007. una geometría de pintores"33. A pesar de los elementos del cubismo analítico que habían entrado en el trabajo de Gris en la preguerra, sus pinturas también compartían numerosos aspectos teóricos expresados en la teoría del cubismo de Puteaux.

Posteriormente, en octubre de 1919, los artistas Gleizes, Braque, Férat, Léger, Archipenko, Marcoussis y Survage crearon una segunda Section d'Or ${ }^{34}$ con el fin de dar a conocer las obras de otros artistas innovadores, realizando un año más tarde una nueva colectiva en la Galerie La Boétie.

\section{JUAN GRIS Y SU RELACIÓN CON LA SECTION D'OR}

Los encuentros comenzaron a realizarse los sábados por la tarde durante 1911 en Puteaux, a las afueras de París, donde Jacques Villon tenía su estudio. Allí se solían dar cita, entre otros, los hermanos Duchamp, Juan Gris, Gleizes, Metzinger, Picabia, Léger, La Fresnaye, así como los poetas Paul Fort, Ribemont-Dessaignes, Jean Cocteau y Joachim Gasquet. Tal y como comenta la investigadora Lynda Dalrymple Henderson, "el grupo resultante de los artistas de Puteaux, como se les solía llamar, mantuvieron contacto con Mercereau, sus amigos poetas del grupo Abbaye de Créteil, y el círculo simbolista Closerie des Lilas. También fueron incluidos Guillaume Apollinaire y Maurice Princet en sus reuniones, con artistas como Juan Gris, Marie Laurencin y Alexander Archipenko"35. A diferencia de Metzinger, Gris no dejó grandes registros de las lecciones que pudo tomar de Princet, a pesar de que tenía una sólida formación en matemáticas y física, de hecho Gris estaba muy bien preparado para explorar las nuevas

${ }^{33}$ J. METZINGER, Le cubisme etait né: Souvenirs, Paris, 1972, pp. 62-63. También remitirse al libro de L.D. HENDERSON, The Fourth Dimension and Non-Euclidean Geometry in Modern Art, Princeton, 1983, p. 70.

${ }^{34}$ Para recabar más información, remitirse a la siguiente página web: http://www.rij-rousseau.de/golden_section/golden_section.html, consultado el 15 de noviembre de 2015 .

${ }^{35}$ L.D. HENDERSON, Op. cit., p. 66. 


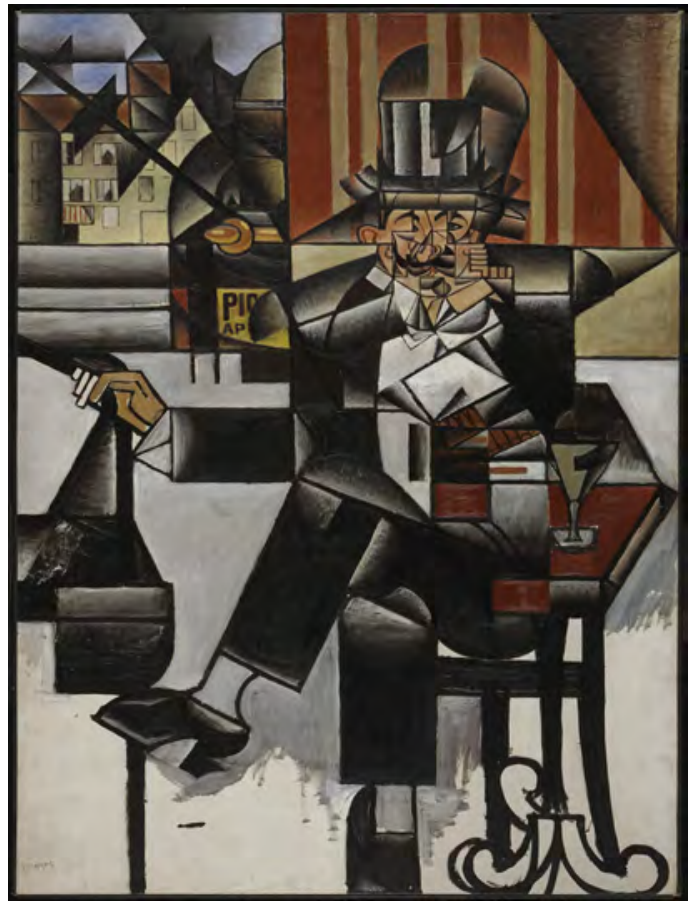

- Fig. 2. Juan Gris. Hombre en el Café. 1912. Philadelphia Museum of Art http://commons.wikimedia.org/wiki/File:Juan_Gris_-_Man_in_a_ Caf\%C3\%A9.jpg.

geometrías en el arte. Pero, en cualquier caso, solo podemos especular sobre las posibles asociaciones tetradimensionales de sus pinturas, teniendo en cuenta la teoría del cubismo de Puteaux y las ideas contemporáneas de la cuarta dimensión. En este sentido, se podrían señalar los siguientes trabajos en relación con una aplicación más matemática de su pintura: Hombre en el Café (Fig. 2), donde se observa una clara composición de red lineal, y Reloj y Botella (Fig. 3), ambas de 1912, trabajos que pudieron estar influenciados por Metzinger, cuando eran alumnos de Maurice Princet.

Otra posible conexión con el tema de la cuarta dimensión serían sus trabajos de 1913, por ejemplo Paisaje en Céret (Fig. 4), de hecho Gris había encontrado la manera de representar una mayor complejidad espacial en sus trabajos de 1913-1914. El acercamiento de Juan Gris hacia unas consideraciones matemático-filosóficas resultan evidentes durante estos años, de hecho, Lynda Dalrymple Henderson cree que "Metzinger y Gris, alumnos de Princet, fueron capaces de proporcionar a Ozenfant una explicación directa del sentido del término de la cuarta dimensión en la teoría cubista de preguerra" ${ }^{\prime 36}$. Por otra parte, tampoco podemos olvidar el cuadro Still Life (1917), donde se "expresa una esencia platónica, alejada de todos los accidentes diarios, la realidad perceptiva. El color local está separado de formas individuales y funciona en la composición como un nivel distinto desde su función meramente descriptiva" ${ }^{\prime \prime 3}$. De acuerdo con Lionello Venturi, Juan Gris daba valor a la regla que corrige lo emocional, defendiendo que el noúmeno precede al propio fenómeno. André Lhote ha comentado que en este periodo Gris hablaba sólo del noúmeno y se alejaba de cualquier destello de manifestación accidental de la realidad, siendo las sombras negras que aparecen en las composiciones de Gris signos noumenales y no recuerdos perceptivos ${ }^{38}$.

Por otro lado, si retomamos el apartado que se hace de la terminología relacionada con la arquitectura en la obra de Juan Gris, de acuerdo con Agustín Sánchez, se produce una interpretación insuficiente: "cobran un alcance meridianamente claro si se ubican en un contexto lulista y herreriano como el Discurso de la figura cúbica. Si así se hace, debe entenderse por arquitectura no la más estricta y gremial acepción de la palabra, sino una mucho más amplia y ambiciosa: nada menos que la transcripción a la medida humana de los cánones y módulos que estructuran el universo, convirtiéndolo en un cosmos inteligible. $Y$, en consecuencia, trasladable por igual a edificio o lienzo, músico o poema, o cualesquiera de las restantes manifestaciones del espíritu que tienen cabida en nuestro orbe, y que de ese modo alentaría desde su original estatuto creador hasta aflorar en el arte bajo la organización y armonía de las formas" ${ }^{\prime 39}$.

A pesar de este supuesto interés por las matemáticas, que mostraban todos los parti-

${ }^{36}$ Ibídem, p. 302.

${ }^{37}$ Ibídem, p. 308.

${ }^{38}$ L. VENTURI, History of Art Criticism, New York, 1964, pp. 297-298.

39 A. SÁNCHEZ VIDAL, "Eminencia Gris", en AA.VV., Juan Gris. Pinturas..., p. 134. 


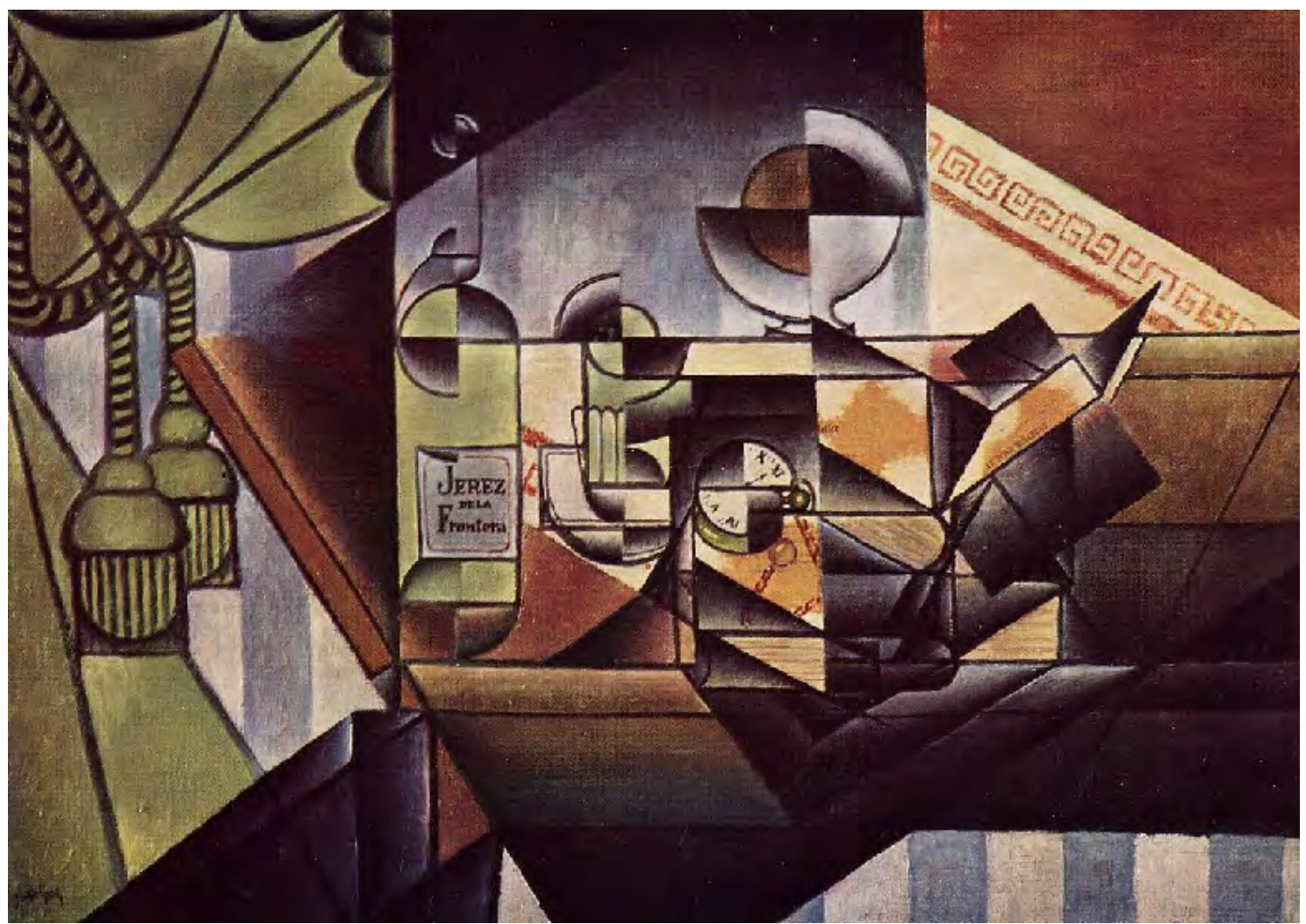

- Fig. 3. Juan Gris. Reloj y botella. 1912. Colección privada. http://es.wahooart.com/a55a04/w.nsf/Opra/ BRUE-6WHL84.

cipantes de la primera exposición, Juan Gris sería el único artista admitido en 1912 en la Section d'Or que realmente tenía un verdadero interés por las proporciones matemáticas de la sección dorada a la hora de construir sus composiciones, tal y como se puede observar en el complejo sistema de enrejados y formas geométricas que componían sus trabajos; sirva de ejemplo su trabajo Hombre en el Café (Fig. 2), ya anotado anteriormente. En este sentido, podrían resultar muy escasos en número los cuadros expuestos en 1912 que muestren las teorías de proporción divina, la cuarta dimensión o consideraciones de matemáticas de proyección más espiritual, a excepción del Portrait de M. Robert G. (1910) de Gleizes, La Jeune femme (1912) de Jacques Villon y los trabajos de Juan Gris, que de acuerdo con las palabras de Lynda Dalrymple Henderson: "En este periodo del cubismo sintético, de unas formas fuertemente definidas y orientación claramente bidimensional, sólo un único miembro del grupo Puteaux se sintió atraído hacia la cuarta dimensión: Juan Gris" ${ }^{40}$. En definitiva, cada artista intentó abrir nuevas perspectivas al cubismo picassiano, aspecto que era asumido por el grupo como una losa impositiva.

Se ha discutido mucho sobre si el estilo de cuadrículas - enrejado de Gris estaba en conexión con los intereses de la proporción de la sección aurea. De hecho, Gris había explorado esta práctica del cubismo analítico a principios de 1912, caso de Guitar and Flowers (Fig. 5), donde se da el uso de múltiples perspectivas en línea con la teoría del cubismo de Puteaux. En cualquier caso, su mayor interés se orientaría hacia el cubismo sintético, tal y como lo expresa el propio artista: "Digo que es deductivo porque las relaciones pictóricas entre las formas coloreadas me sugieren ciertas relaciones particulares entre elementos de una realidad imaginativa. La matemática pictórica me conduce a la física representativa. La calidad o la dimensión de una forma o de un color me

${ }^{40}$ L.D. HENDERSON, Op. cit., p. 74. 


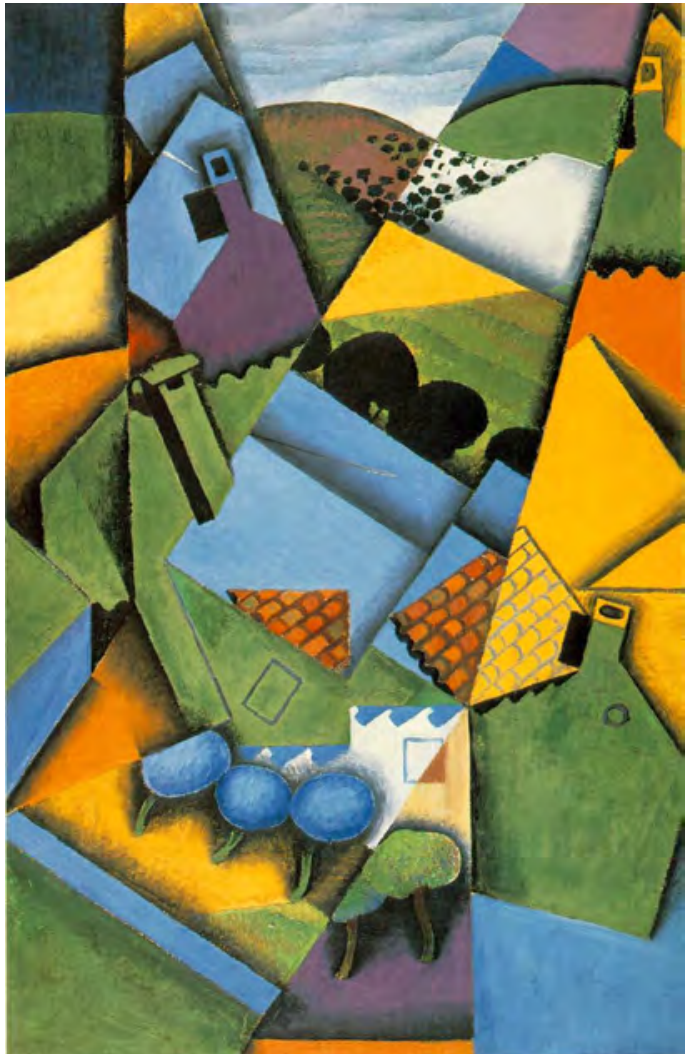

- Fig. 4. Juan Gris. Paisaje en Ceret. 1913. Colección privada.http://paintingdb.com/s/9985/.

sugieren la denominación o el adjetivo de un objeto. Por esto yo no conozco jamás por adelantado el aspecto de un objeto representado. Si particularizo las relaciones pictóricas hasta la representación de objetos, es para evitar que el espectador de un cuadro lo haga por sí mismo y que el conjunto de formas coloreadas le sugiera una realidad no prevista por mí" ${ }^{\prime \prime 1}$.

A pesar de las diferentes dudas suscitadas, el pintor madrileño será el que más se va adentrar en los entresijos de una geometría de corte más espiritual, debido a su formación matemática, y al haberse nutrido en París de la influencia de personajes con altos conocimientos en metafísica y estética de las matemáticas. Para algunos autores, "el pensamiento de Juan Gris se encuentra en tres contextos: el arte, por supuesto, pero también la filosofía y especialmente las Ciencias y las matemá-

${ }^{41}$ D-H. KAHNWEILER, Juan Gris. Vida y pintura, Madrid, 1971, p. 328. Texto del artista publicado por primera vez en Der Querschnitt, no 1-2, 1923, pp. 77-78.

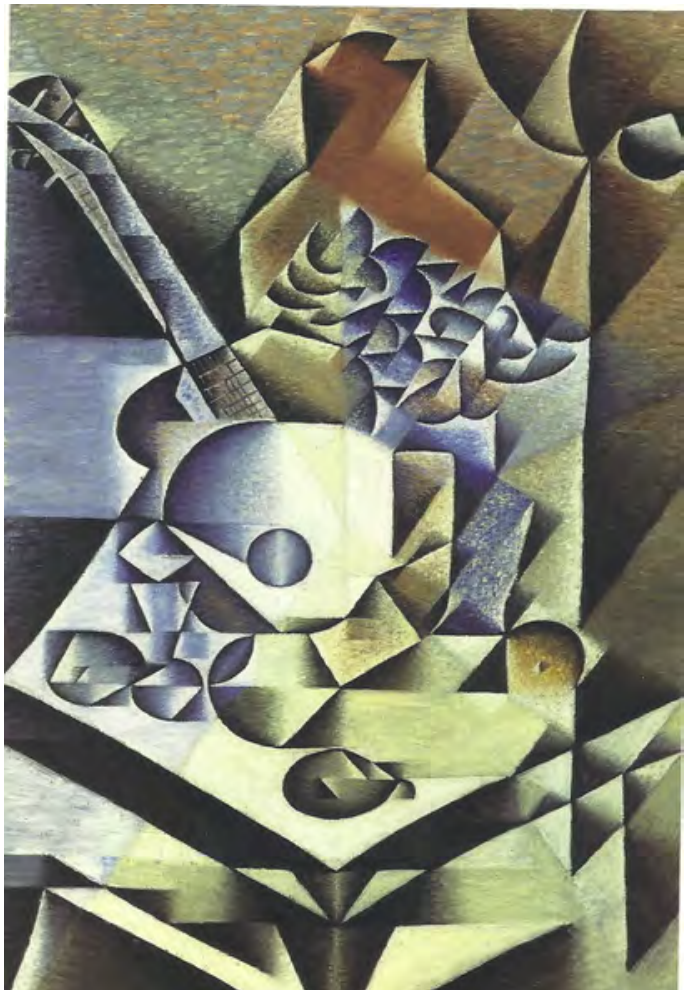

- Fig. 5. Juan Gris. Guitarra y flores. 1912. AA.VV., Juan Gris. Paintings and Drawings 1910-1927. Catálogo de la Exposición, del Museo Nacional Centro de Arte Reina Sofía, Madrid, 2005.

ticas. Para denotar las constantes de la construcción pictórica, él habla de pintura matemática. En cuanto al diseño general de la mesa - su arquitectura, como él dice - la compara con la síntesis de un compuesto químico"42. De hecho, también William A. Camfield ${ }^{43}$ recuerda que uno de estos artistas, Lhote, afirmaría más tarde que Gris utilizaba la sección áurea en sus pinturas ${ }^{44}$. André Lhote ${ }^{45}$, en su Trai-

${ }^{42}$ C. GREEN, “L'idée de Juan Gris", en AA.VV., Juan Gris. Peintures et dessins. 1887-1927, Marseille, 1999, p. 23.

43 W.A. CAMFIELD, "Juan Gris and the golden section", Art Bulletin, 47, March 1965, pp. 128-134.

${ }^{44}$ Esta opinión es igualmente corroborada por D. COTTINGTON, Op. cit., p. 112; P. BALL, Shapes: Nature's Patterns: a Tapestry in Three Parts, New York, 2009, p. 239; R. STANLEY JOHNSON, Cubism \& la Section D'or: Works on Paper, 1907-1922: Selections from a Private Collection, Chicago, 1989, p. 13; D. JOHNSON y T. MOWRY, Mathematics: A Practical Odyssey, Belmont, 2012, p. 518.

${ }^{45}$ A. LHOTE, Traités du Paysage et de la Figure, Paris, 1958. 


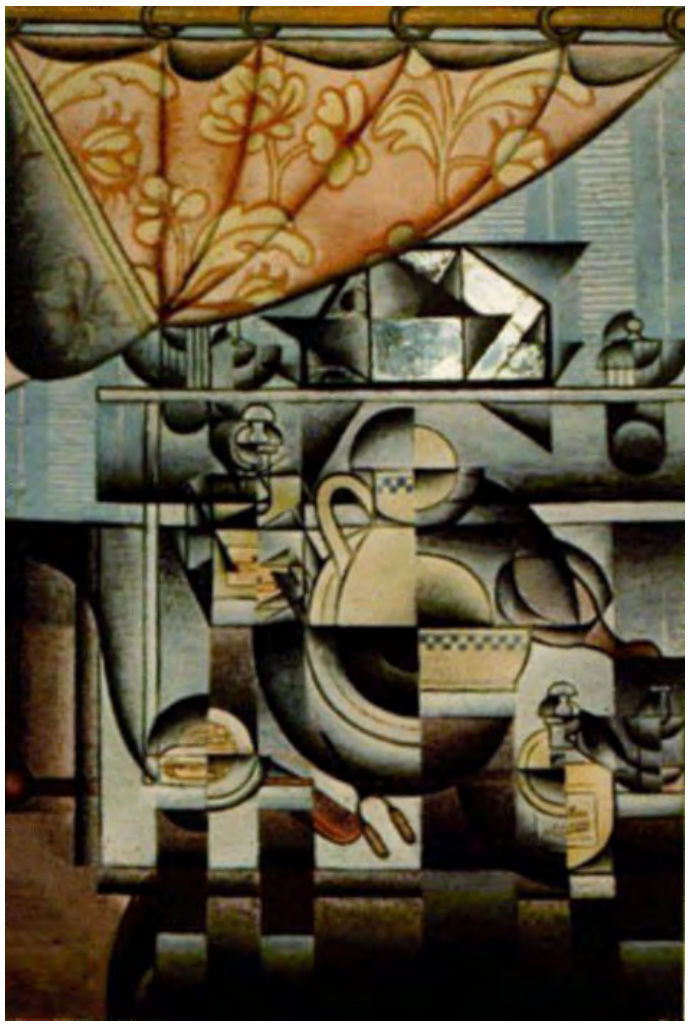

- Fig. 6. Juan Gris. El Lavabo. 1912. Collection Vicomtesse de Noailles, Paris. http://www. ibiblio.org/wm/paint/auth/gris/gris.lavabo. jpg.

tés du Paysage et de la Figure, escribió que los cubistas, en particular Juan Gris, construían sus cuadros basándose en la sección áurea. En palabras de R. Stanley Johnson, "Gris fue considerado el más puro cubista y uno de los que llevó el movimiento a su más alto nivel...... Gris a menudo inició sus trabajos con una concepción figurativa científica y abstractarizada basada en el uso de la Section d'Or, una fórmula que se relaciona de manera cercana con el concepto más general de Kahnweiler sobre la estructura esquelética"46. Por otro parte, para Agustín Sánchez Vidal, "aunque nos queda mucho por saber sobre cómo, dónde y con qué alcance obtuvo Juan Gris su muy elaborada doctrina, cabe proponerle como un eslabón más en ese encadenado que forman Ramón Llull y Juan de Herrera. La teoría o desarrollo del cubismo del pintor madrileño

${ }^{46}$ R. STANLEY JOHNSON, Cubism \& La Section d'Or: Reflections on the Development of the Cubist Epoch 1907-1922, Chicago-Düsseldorf, 1991, pp. 20-21. viene a ser una de las prolongaciones plausibles del Discurso de la figura cúbica" ${ }^{47}$.

Entre las nueve pinturas y tres dibujos que expuso Gris para la exposición de $L a$ Séction d'Or, destacan los lienzos Hombre en el café (Fig. 2), El lavabo ${ }^{48}$ (Fig. 6) y Reloj y botella (Fig. 3) (estando las tres fechadas en 1912), donde se podría dar el uso de la sección áurea. No obstante, esta idea ha sido rechazada en los estudios de Roger Fischler ${ }^{49}$, donde se afirma que a pesar de conocer dicha metodología matemática, no llegó a emplearla de una manera muy clara. Kanhweiler se refiere a Gris en estos términos: "Su punto de partida -cosa que parecerá extraña a quien no vea en Gris más que un lógico- se situaba en la penumbra del subconsciente. Una proporción, tal como, por ejemplo, la Section d'Or, no era para él trampolín desde el que se lanzaba su imaginación hacia una Arquitectura coloreada abstracta o que, al menos, le parecía de esta manera"50. En cualquier caso, para este anterior autor como para Douglas Cooper, Gris sí que influye sobre algunos de los participantes (Gleizes, Metzinger y Marcoussis) al emplear la cuadrícula y la firme estructura lineal de sus composiciones. Si en la pintura Hombre en el café (Fig. 2), se utiliza una rigurosa cuadrícula para ordenar la pintura, en Reloj y botella (Fig. 3), se aprecian rectángulos y cuadrados de la Sección de Oro como receptáculo de títulos de poemas de Apollinaire, que iban a anunciar la presencia futura del collage. Otras propuestas que también pueden mostrar la proporción divina y se mostraron en dicha exposición fueron los dibujos La Guitare (1912) y Cabeza de mujer ${ }^{51}$ (Fig. 7). Para Juan Gris: “Un cuadro es una síntesis, como sintética es toda arquitectura.

\footnotetext{
${ }^{47}$ A. SÁNCHEZ VIDAL, Op. cit., p. 132.

${ }^{48}$ D. COTTINGTON, Op. cit., p. 150.

${ }^{49}$ R. HERTZ-FISCHLER, “Juan Gris, son milieu et le 'nombre d'or'", Canadian Art Review, 7, 1980, pp. 33-36; ÍDEM, “Le nombre d'or en France de 1896 à 1927", La Revue de l'art, 118, no 4, 1997, pp. 9-16.
}

\footnotetext{
${ }^{50}$ D-H. KAHNWEILER, Op. cit., p. 266.

${ }^{51}$ P. ESTEBAN LEAL, Op. cit., pp. 32-33.
} 


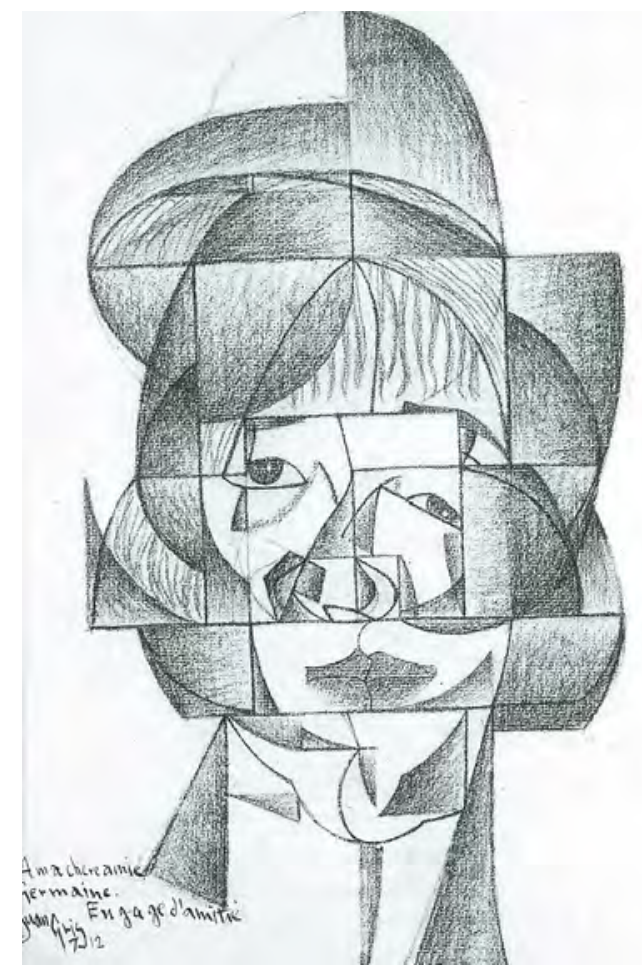

- Fig. 7. Juan Gris. Cabeza de mujer. Estudio para el retrato de Germaine Raynal. 1912. Colección privada.

La estética ha analizado el mundo pictórico y nos ha proporcionado los elementos. Es evidente que esos elementos se encarnan al sustituir a las formas abstractas que componen el cuadro como los cuerpos simples del hidrógeno y el oxígeno no son sustituidos por la formula $\mathrm{H}_{2} \mathrm{O}$ para realizar la síntesis del agua" 52 .

Cuando Gris retrata en 1912 a la esposa de Maurice Raynal, bien sea al óleo (Fig. 8) o en el dibujo Cabeza de mujer (Fig. 7), su nuevo método de estructuración compositiva ya estaba desarrollado, de hecho, "se aprecia claramente en estas obras dedicadas a Mme. Raynal como la escena se ha dividido en una rejilla o cuadrícula en la que cada compartimento contiene uno de los aspectos del rostro de la modelo, abordado desde un punto de vista diferente, mientras que el conjunto incluye tanto las vistas de frente como las de perfil, e incluso, como ocurre en el caso de 43.

${ }^{52} \mathrm{~J}$. GRIS, Sobre las posibilidades de la pintura..., p.

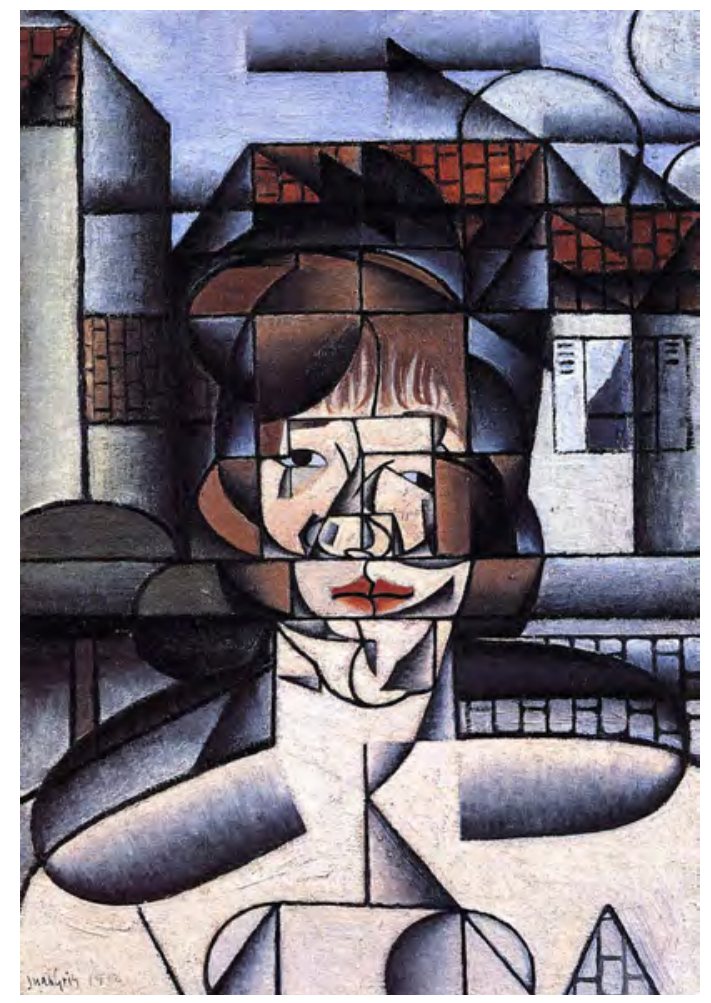

- Fig. 8. Juan Gris. Retrato de Germaine Raynal. 1912. Colección privada. http://www.wikiart. org/en/juan-gris/portrait-of-germaine-raynal-1912.

la representación de la nariz, incorpora la perspectiva di sotto in sù. Con el fin de unificar los distintos elementos de la escena, Gris llega a fusionar uno de los hombros de la protagonista con el adoquinado de la calle que actúa como fondo del retrato.....el uso y el perfeccionamiento de esta especial estructuración compositiva de acuerdo con una cuadrícula o rejilla previa no es casual, sino que está estrechamente relacionado con los principios de la Section d'Or o Sección Áurea. El Salón de la Section d'Or se cuenta entre las exposiciones de arte y publicaciones de 1912 que hacen de ese año un referente del arte moderno" 53 .

El uso de los lenguajes matemáticos se aplicó también al campo de la escultura, de hecho Jacques Lipchitz, miembro de la logia masónica Voltaire, al igual que Juan Gris, ayudó a éste en la construcción de la escultura titulada Arlequín (Fig. 9) de

\footnotetext{
${ }^{53}$ P. ESTEBAN LEAL, Op. cit., pp. 32-33.
} 


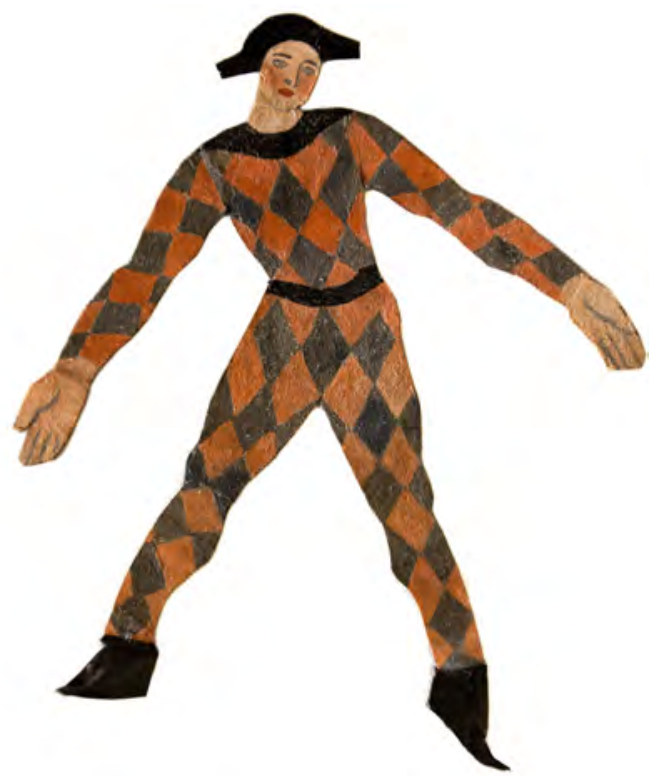

- Fig. 9. Juan Gris. Arlequín. 1923. Museo Nacional Centro de Arte Reina Sofía. http://www. museoreinasofia.es/sites/default/files/descargas/juan_gris-arlequin_0.jpg.

1923, en donde los dos artistas emplearon el triángulo de Kepler, que está basado en la proporción divina para obtener las proporciones deseadas. Igualmente, bajo esta intencionalidad de proyección matemática, debemos mencionar Guitarra y por otro lado Naturaleza muerta con copa y damero, ambas de 1913. Durante este año, el artista cambia la estructuración compositiva en forma de rejilla por un sistema de bandas, que en un principio son verticales, pero que posteriormente asumen posiciones oblicuas, triangulares y en abanico. Para Gris, "la representación de ese mundo sustancial (y digo sustancial porque considero las nociones de objetos como sustantivos), puede dar lugar a una estética, a una elección de elementos que sólo son propios para desvelar ese mundo de nociones que existe puramente en el espíritu". En esta misma línea, el pintor español añade lo siguiente: “De todo esto se deduce que como no considero el cubismo como un procedimiento, sino como una estética e incluso como un estado de espíritu, forzosamente tiene que haber una correlación con todas las manifestaciones del pensamiento con- temporáneo. Uno puede inventar aisladamente una técnica, un procedimiento, pero uno no inventa todas las piezas de un estado de espíritu" ${ }^{54}$. De hecho, continuando con las declaraciones del artista, debemos emplazar lo siguiente: "En todas las grandes épocas del arte, se siente la necesidad de representación de un mundo sustancial y espiritual. Cada época lo ha influido y diversificado según sus exigencias e inquietudes" ${ }^{\prime 55}$.

\section{CONCLUSIONES}

La participación de Juan Gris en las diferentes reuniones y encuentros que se mantuvieron dentro del Groupe de Puteaux le permitieron reflexionar sobre otras orientaciones técnicas a la hora de abordar el potencial creativo del cubismo. No sólo su interés por el cubismo sintético, sino su acercamiento a pautas de sistemas de enrejados, claramente articulados bajo premisas matemáticas, le permitió distinguirse dentro del grupo. Su anterior formación técnico-científica le facilitó el aprendizaje y la aplicación de pautas matemáticas expuestas por Maurice Princet en las diferentes sesiones que mantuvo junto a otros cubistas. No obstante, son numerosas las dudas suscitadas en torno a si realmente hubo una verdadera aplicación de la sección aurea, por ello, a modo de hipótesis se barajan diversas obras como las más cercanas a esta sintonía matemática. A pesar de realizar un trabajo técnico, Juan Gris tuvo una proyección humanista y ciertamente espiritual en lo que se refiere a la búsqueda de un cubismo que estuviera integrado por pautas y constantes, como era la posible aplicación matemática en base a la proporción divina, con el propósito de generar un cubismo espiritual y alejado de la habitual visión mecanicista.

${ }^{54}$ J. GRIS, Correspondencia......, p. 403. Extraído de una respuesta a una encuesta a Bulletin de la vie artistique, finales de 1924.

${ }^{55}$ ÍDEM, Sobre las posibilidades de la pintura..., p. 